Mini-Review article

\title{
Cotrimoxazole and teicoplanin in the management of Covid-19: Pleiotropic effects, shadows and lights
}

\author{
Hayder M. Al-Kuraishy*, Ali I. Al-Gareeb and Ali K. Al-Buhadily \\ Department of Clinical Pharmacology, Medicine and Therapeutics, College of Medicine, Al-Mustansiriya \\ University, P.O. Box 14132, Baghdad, Iraq
}

*Corresponding author. E-mail: hayderm36@yahoo.com; Phone: +96-407906230487

\section{Article history \\ Keywords \\ Coronavirus Cotrimoxazole \\ Covid-19 \\ Teicoplanin}

Received : October 20, 2020

Accepted : October 26, 2020

\begin{abstract}
At present, coronavirus disease 2019 (Covid-19), caused by SARS-CoV-2 infection, has turned into a global pandemic. Comparable to the H7N9 virus, SARS-CoV-2 causes soaring mortality in elderly patients who have pre-existing chronic diseases. SARS-CoV-2 infects human epithelial cells by binding to human angiotensinconverting enzyme2 (ACE2). Multi-organ failure has been observed in some patients with severe COVID-19. TheACE2 expression profile suggests that SARS-CoV-2 infection may initiate or even directly cause organ failure. In addition to direct infection, evidence shows that an elevated inflammatory immune response is also involved in the pathogenesis of SARS-CoV-2 infection. The immunomodulatory and anti-inflammatory activity of the cotrimoxazole (CTX) and teicoplanin are seen in several studies that show the lower concentrations of plasma pro-inflammatory markers like CRP, IL6 in continuous CTX prophylaxis, suggesting its role as antiinflammatory and immunomodulation. The present review concludes that both teicoplanin and CTX are effective in the management of Covid-19 through anti-SARSCoV-2 and anti-inflammatory effects as well as controlling of secondary bacterial infections.
\end{abstract}

(c) 2020 Global SciTech Ocean Publishing Co. All rights reserved. ISSN. 2581-5008

\section{INTRODUCTION}

In current decades, the world has veteran outbreaks of newly talented viruses, including severe acute respiratory syndrome coronavirus (SARS-CoV-1), Middle East respiratory syndrome coronavirus (MERS-CoV), H5Nl virus, pandemic $\mathrm{H} 1 \mathrm{Nl}$ virus, and H7N9 virus (Al-Kuraishy et al., 2020a). At present, coronavirus disease 2019 (COVID-19), caused by SARS-CoV-2 infection, has turned into a global pandemic. Comparable to the H7N9 virus, SARS-CoV-2 causes soaring mortality in elderly patients who have preexisting chronic diseases.

Acute lung injury (ALI) is one of the main causes of the high mortality of patients infected with H5N1, H7N9, MERS-CoV, SARS-CoV-1, and SARS-CoV-2 (Al-Kuraishy et al., 2020b). Increasing evidence suggests that an elevated inflammatory immune response and cytokine storms are connected to ALI and fatality caused by these viruses (Al-Kuraishy et al., 2020c).

Up till now, there is no elective treatment for ALI caused by viral infections. Therefore, this study reviews the immune pathogenesis of SARS-CoV-2 infection and argues the possibility of using antibiotics as a drug for extenuating the severity of COVID-19 illness.

\section{SARS-CoV-2 INFECTION \& IMIMUNE RESPONSE}

SARS-CoV-2 infects human epithelial cells by binding to human angiotensin-converting enzyme2 (ACE2) (Fig. 1). ACE2 gene is expressed in cell clusters in organs including the lung, heart, oesophagus, kidney, bladder, testis and ileum, indicating that these organs are at high risk of SARS-CoV-2 infection (Al-Kuraishy et al., 2020d). Constantly, multiorgan failure has been observed in some patients with severe COVID-19.

The ACE2 expression profile suggests that SARS-CoV-2 infection may initiate or even directly cause organ failure (Al-Kuraishy et al., 2020e). In addition to direct infection, evidence shows that an elevated inflammatory immune response is also involved in the pathogenesis of SARS-CoV-2 infection (COVID-19) (Al-Kuraishy and Al-Gareeb, 2020). 


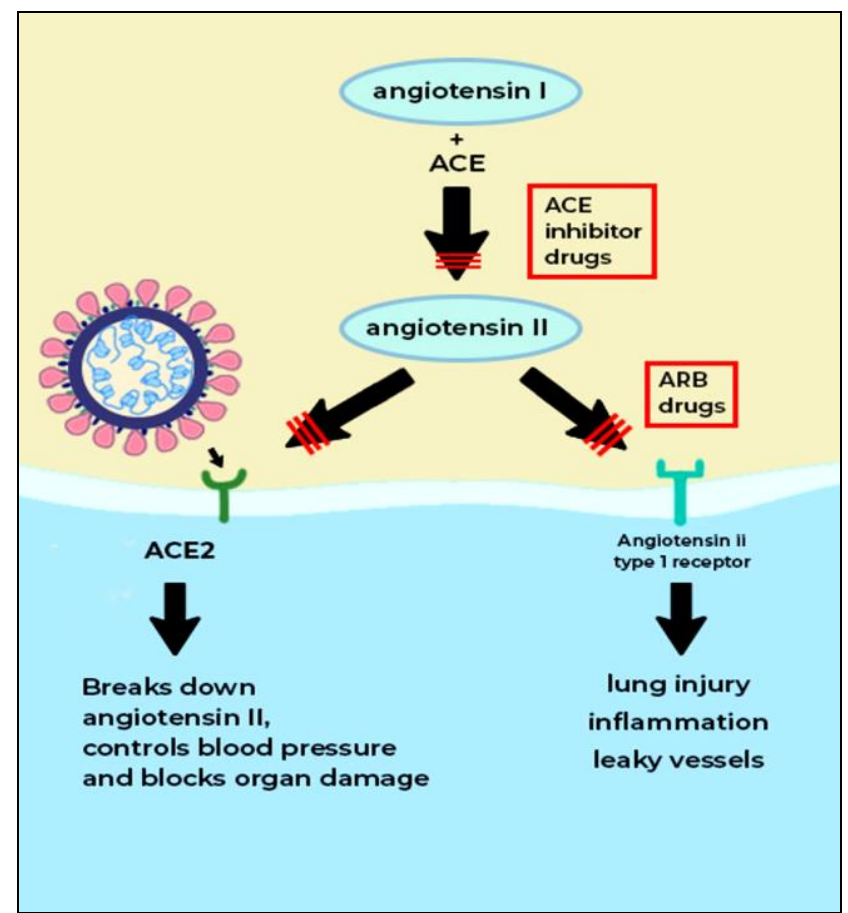

Fig. 1. SARS-CoV-2 and ACE2 receptors

Patients in the intensive care unit (ICU) had higher plasma levels of IL-2, IL-7, IL-10, G-CSF, IP10, MCP-1, MIP-1 $\alpha$, and TNF $\alpha$ than non-ICU patients. Some severe patients also had elevated IL-6 levels in their plasma (which correlates with respiratory failure and fatality. Besides, the serum levels of C-reactive protein, which is positively regulated by IL-6, are elevated in patients with severe COVID-19 (Al-Kuraishy et al., 2020f). The systemic elevations of cytokines are reminiscent of the cytokine storm or cytokine release syndrome (CRS) that occurs in severe infections of influenza viruses, MERS-CoV, and SARS-CoV-1. Due to the critical role of IL-6 in promoting CRS, inhibition of IL-6 or IL-6 receptor with antibodies has been proposed to treat patients with severe COVID-19. How SARS-CoV-2 infection triggers cytokine storms remains largely speculative. As well, elevated cytokines in patients with severe COVID-19 linked with marked lymphopenia. A recent autopsy study of patients with COVID-19 shows that SARS-CoV-2 infection is detected in CD169+ macrophages that express the ACE2 molecule and produce IL- 6 . The infection of these CD169+ macrophages correlated with lymphocyte apoptosis in the spleen and lymph nodes. These findings may partly explain the excessive inflammation and lymphopenia during severe COVID-19 (Al-Kuraishy et al., 2020g).

High neutrophil counts are significantly associated with COVID-19 fatality and the neutrophil-to-lymphocyte ratio is an independent risk factor for the illness severity of COVIDI9. When a cytokine storm occurs, chemokines recruit neutrophils to lung lesions, and pro-inflammatory cytokines, such as IL-1 $\beta$ and IL-6, activate neutrophils to produce NETs, which may contribute to ALI and mortality in patients with COVID-19 (Fig. 2) (Zhang et al., 2020).

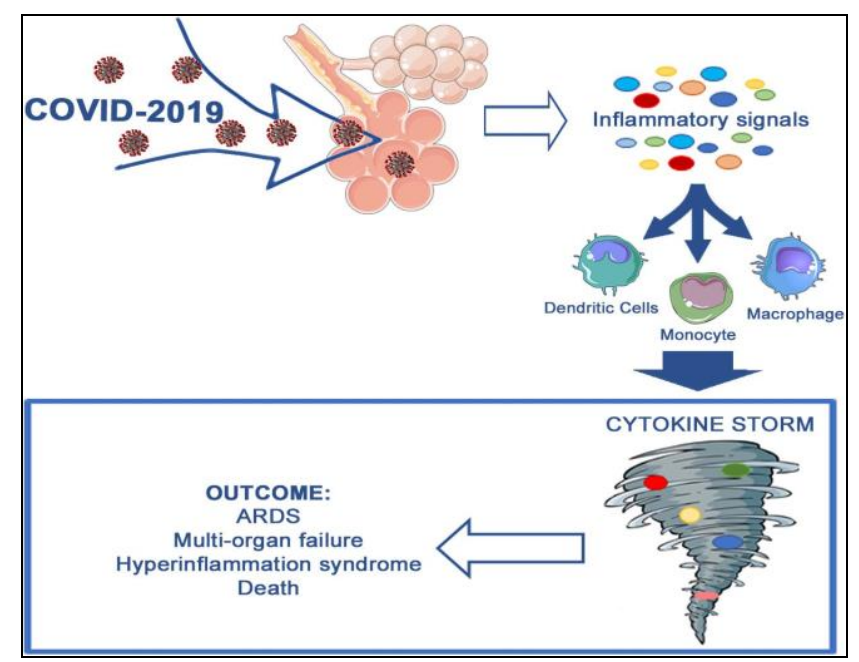

Fig. 2. Covid-19 and cytokine storm

\section{TRIMETHOPRIM AND COTRIMOXAZOLE}

Cotrimoxazole (CTX) is an effective broadspectrum antibiotic with antifungal, antiprotozoal, activity. The rationale behind the use of cotrimoxazole is its anti-inflammatory and immunomodulatory action. The mortality among the COVID19 patients is mainly due to the acute respiratory distress syndrome (ARDS) or ALI, pulmonary embolism and respiratory failure mediated by cytokine storm due to unopposed multiplication of cascade of inflammatory mediators (Al-Kuraishy et al., 2018).

The immunomodulatory and anti-inflammatory activity of the CTX is seen in several studies that show the lower concentrations of plasma proinflammatory markers like CRP, IL6 in continuous CTX prophylaxis, suggesting its role as antiinflammatory and immunomodulation (Choudhari et al., 2020). The responsibility of IL6 and TNF- $\alpha$ in the pathogenesis of COVID-19 mortality is well recognized and the role of CTX in the suppression of TNF $\alpha$ is also recognized (Bourke et al., 2019).

Lymphopenia is associated with adverse outcomes in COVID-19, and CTX has shown an increase in lymphocyte count in short and long therapy duration, but these study findings are not dependable; however, no important impact of CTX was seen on immune activation of CD8 T cells (Khalil et al., 2019). Oxidative stress has an important aspect of the cytokine storm, which is also reduced by CTX (Gough et al., 2020).

ARDS is one of the life-threatening complications of COVID-19 caused by the body's hyper-immune response to the virus in the form of a cytokine storm syndrome (CSS). The CSS occurs as a result of neutrophil recruitment into the lung due to the stimulation of the formyl peptide receptors (FPRs) by Damage Associated Molecular Patterns (DAMPs) which are released upon mitochondrial injury of host cells (Bourke and Prendergast, 2020). FPRs are situated on the outer surface of the cell membrane of the neutrophils and monocytes, and when stimulated cause the release 
of intracellular and extracellular reactive oxygen series (ROS) which can drive the cytokine activation and stimulate the formation of Neutrophil Extracellular Traps (NETs) which block the alveolar capillary bed leading to hypoxaemia (Fig. 3) (Quadery et al., 2020a).

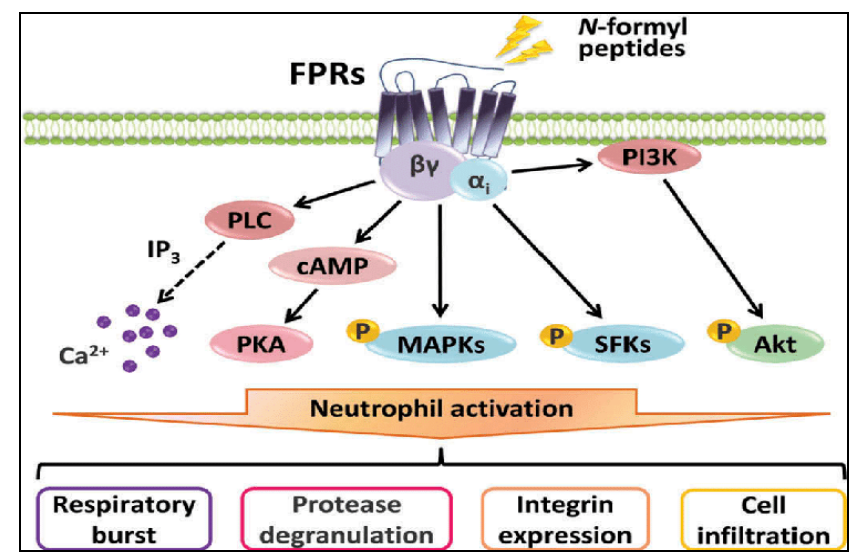

Fig. 3. Formyl peptide receptors and inflammations

CTX blocks the FPR's and can reduce the movement of neutrophils to the lung, generation of ROS, production of pro-inflammatory cytokines and formation of ALI. Published data shows that CTX has anti-cytokine effects reducing interleukin-1, 2, 6,8 and TNF- $\alpha$ production (Quadery et al., 2020b). Several of these cytokines are shown to be raised in the cytokine storm associated with COVID-19. This offers a possible explanation for the observed clinical benefit by reducing neutrophil, monocyte and lymphocyte activation leading to a reduction in the risk of ARDS (Park et al., 2020).

Although both dapsone and CTX have effects upon the immune system which were first described for dapsone over 50 years ago. Both drugs share the same sulphonamide ring with similar antibacterial effects, with detailed studies of dapsone showing a dose-dependent reduction in the generation of tissue-damaging 'oxygen free radicals' from neutrophils (Al-Kuraishy, 2017a).

This reduction results from dapsone's ability to block stimulation of the formyl peptide receptors (FPR), which are abundantly expressed on the surface of neutrophils and monocytes. Their activation drives the production of both intracellular and extracellular oxygen free radical release leading to further inflammatory cytokine production (Al-Kuraishy and Al-Gareeb, 2020; AlKuraishy et al., 2020g). The blockade of FPR by dapsone and CTX herefore has marked antiinflammatory effects (Al-Kuraishy, 2016).

CTX reduces ALI in patients with severe COVID-19, thereby reducing the need for ventilatory support and improving outcomes. This drug has no direct anti-viral effects but may present protection against ARDS. The valuable effect of CTX is apparent within hours of the first dose, likely reflecting their excellent absorption and lung penetration. Appropriate gratitude of any clinical deterioration from the underlying cytokine storm syndrome is important and delayed treatment, may reduce the ability of these drugs to act before the blockade of the alveolar capillary bed by neutrophils, since the risk of profound hypoxemia may be difficult to reverse (Al-Kuraishy, 2017b).

Besides, CTX has anti-cytokine effects reducing interleukin-1, 2, 6, 7, 8 and TNF- $\alpha$ production. Several of these cytokines are involved in the cytokine storm of COVID-19. This offers a possible explanation for the observed clinical benefit by reducing neutrophil, monocyte and lymphocyte activation leading to a reduction in the risk of ARDS as in other viral infections (Fig. 4) (Al-Kuraishy et al., 2019).

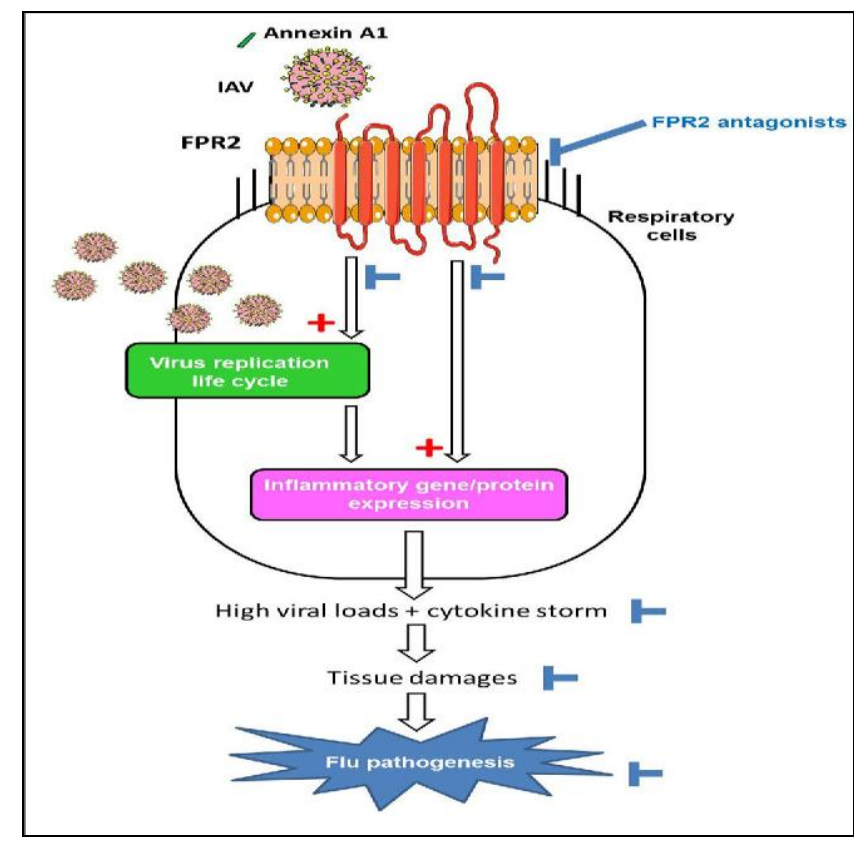

Fig. 4. Formyl peptide receptors and viral pathogenesis

Trimethoprim or CTX are inexpensive drugs licensed for use in respiratory infections with few serious side effects. They are generally available worldwide and may have benefit in preventing ALI in this pandemic. CTX may have advantages over trimethoprim due to the additional immune effects of sulphamethoxazole along with an intravenous preparation for use in deteriorating patients. Reducing ARDS and oxygen demand may be vital to saving lives in countries where healthcare may be easily overwhelmed. Thus, we recommend that these observations should be tested in clinical trials of TMP or CTX in the management of severe COVID-19 (Kadhim et al., 2019).

\section{TEICOPLANIN}

Teicoplanin, a glycopeptide antibiotic routinely used to treat bacterial infection was found to be active in vitro against SARS-CoV, has joined the list of molecules that could be used as a therapeutic arsenal against COVID-19 (Baron et al., 2020). This 
antibiotic, currently used in the treatment of Grampositive bacterial infection, especially in Staphylococcal infections, has already shown efficacy against various viruses such as Ebola, influenza virus, flavivirus, hepatitis $\mathrm{C}$ virus, $\mathrm{HIV}$ and coronavirus such as MERS-CoV and SARS-CoV (Sathyamoorthy et al., 2020). A patent was filed for the treatment of infection caused by MERS-CoV in 2016. In coronaviruses, teicoplanin acts on the early step of the viral life cycle by inhibiting the low $\mathrm{pH}$ cleavage of the viral spike protein by cathepsin $\mathrm{L}$ in the late endosomes thereby preventing the release of genomic viral RNA and the continuation of the virus replication cycle. A recent study by the same authors showed that this activity was conserved on SARS-Cov-2 (the target sequence that serves as cleavage site for cathepsin $\mathrm{L}$ is conserved among SARS-CoV spike protein) (Ceccarelli et al., 2020a). The concentration of teicoplanin required to inhibit $50 \%$ of viruses (IC50) in vitro was $1.66 \mu \mathrm{M}$, which is much lower than the concentration reached in human blood (8.78 $\mu \mathrm{M}$ for a daily dose of $400 \mathrm{mg}$ ) (Jean and Hsueh, 2020).

Moreover, influenza $A$ and $B$ viruses were reported as potential targets of teicoplanin and its chemical derivatives (Ceccarelli et al., 2020b). Newly, additional studies have provided evidence that SARS-CoV-2, similarly to SARS-CoV, is a cathepsin L-dependent virus: in fact, these viruses require a multistep infection process including receptor binding, change in spike (S) glycoprotein confirmation and cathepsin L proteolysis of the $\mathrm{S}$ protein, crucial for virus entry. Teicoplanin was found to specifically inhibit the activity of cathepsin $\mathrm{L}$ and potentially to play a critical role in blocking cell entry of the virus (Petersen et al., 2020).

Based on the aforementioned, teicoplanin has been used either as a potential antiviral agent or as treatment of possible Staphylococcus aureus superinfection in our critical patients with severe SARSCoV-2 pneumonia, since the latter may represent a major complication of respiratory viral infections (Agrawal et al., 2020).

\section{CONCLUSION}

Both teicoplanin and CTX are effective in the management of Covid-19 through anti-SARS-CoV-2 and anti-inflammatory effects as well as controlling of secondary bacterial infections.

\section{Conflict of interest}

The authors declare no conflicts of interest.

\section{REFERENCES}

Agrawal M, Saraf S, Saraf S, Murty US, Kurundkar SB, Roy D, Joshi P, Sable D, Choudhary YK, Kesharwani P, Alexander A (2020). In-line treatments and clinical initiatives to fight against COVID-19 outbreak. Respiratory Medicine, 106192.
Al-Kuraishy HM (2016). Central additive effect of Ginkgo biloba and Rhodiola rosea on psychomotor vigilance task and short-term working memory accuracy. Journal of Intercultural Ethnopharmacology, 5, 7-13.

Al-Kuraishy HM (2017a). Molecular Microbiology and Statins Frontier. Journal of Molecular Microbiology, 1, el02.

Al-Kuraishy HM (2017b). Responsibility of peroxisome proliferator activated receptor- $\alpha$ (PPAR- $\alpha$ ) in epilepsy. Journal of Stroke and Epilepsy, 1, 1-2.

Al-Kuraishy HM, Al-Gareeb AI (2020). From SARS-CoV to nCoV-2019: Ruction and argument. Archives of Clinical Infectious Diseases, 15, el02624.

Al-Kuraishy HM, Al-Gareeb AI, Al-Buhadily AK (2018). Rosuvastatin as forthcoming antibiotic or as adjuvant additive agent: In vitro novel antibacterial study. Journal of Laboratory Physicians, 10, 271-275.

Al-Kuraishy HM, Al-Gareeb AI, Monteiro MC, Al-Saiddy HJ (2020g). Brain injury and SARS-CoV-2 infection: Bidirectional pathways. Current Medical and Drug Research, 4, Art. ID 207.

Al-Kuraishy HM, Al-Gareeb AI, Monteiro MC, Hussein HN (2020f). Covid-19 and Iraq: The exponential epidemic. Journal of Conventional Knowledge and Holistic Health, 4, Art. ID 209.

Al-Kuraishy HM, Al-Gareeb AI, Rasheed HA (2019). Antioxidant and anti-inflammatory effects of curcumin contribute into attenuation of acute gentamicininduced nephrotoxicity in rats. Asian Journal of Pharmaceutical and Clinical Research, 12, 466-468.

Al-Kuraishy HM, Al-Maiahy TJ, Al-Gareeb AI, Musa RA, Ali ZH (2020a). COVID-19 pneumonia in an Iraqi pregnant woman with preterm delivery. Asian Pacific Journal of Reproduction [Epub ahead of print]. Online available http://www.apjr.net/preprintarticle.asp?id=282984.

Al-Kuraishy HM, Al-Naimi, MS, Lungnier, CM, Al-Gareeb AI (2020e). Macrolides and COVID-19: An optimum premise. Biomedical and Biotechnology Research Journal, 4, 189-199.

Al-Kuraishy HM, Al-Niemi MS, Hussain NR, Al-Gareeb AI, Al-Harchan NA, Al-Kurashi AH (2020c). The potential role of renin-angiotensin system (RAS) and dipeptidyl peptidase-4 (DPP-4) in COVID-19: Navigating the uncharted. In: Selected chapters from the reninangiotensin system, Kibel A (Ed). IntechOpen, London, pp. 151-165.

Al-Kuraishy HM, Hussien NR, Al-Naimi MS, Al-Buhadily AK, Al-Gareeb AI, Lungnier CM (2020b). Is ivermectin-azithromycin combination the next step for COVID-19? Biomedical and Biotechnology Research Journal, 4, S101-103.

Al-Kuraishy HM, Hussien NR, Al-Naimil MS, Al-Buhadily AK, Al-Gareeb AI, Lungnier C (2020d). ReninAngiotensin System and fibrinolytic pathway in COVID-19: One-way skepticism. Biomedical and Biotechnology Research Journal, 4, S33-40.

Baron SA, Devaux C, Colson P, Raoult D, Rolain JM (2020). Teicoplanin: an alternative drug for the treatment of COVID-19? International Journal of Antimicrobial Agents, 55, 105944.

Bourke CD, Gough EK, Pimundu G, Shonhai A, Berejena C, Terry L, Baumard L, Choudhry N, Karmali Y, Bwakura-Dangarembizi M, Musiime V, Lutaakome J, Kekitiinwa A, Mutasa K, Szubert AJ, Spyer MJ, Deayton JR, Glass M, Geum HM, Pardieu C, Gibb DM, Klein N, Edens TJ, Walker AS, Manges AR, Prendergast AJ (2019). Cotrimoxazole reduces systemic inflammation in HIV infection by altering the gut microbiome and 
immune activation. Science Translational Medicine, 11, eaav0537.

Bourke CD, Prendergast AJ (2020). The Antiinflammatory Effects of Cotrimoxazole Prophylaxis for People Living With Human Immunodeficiency Virus in Sub-Saharan Africa. The Journal of Infectious Diseases, 222, 347-350.

Ceccarelli G, Alessandri F, d'Ettorre G, Borrazzo C, Spagnolello O, Oliva A, Ruberto F, Mastroianni CM, Pugliese F, Venditti M, Intensive Care COVID-19 Study Group of Sapienza University (2020a). Is teicoplanin a complementary treatment option for COVID-19? The question remains. International Journal of Antimicrobial Agents, 56, 106029.

Ceccarelli G, Alessandri F, Oliva A, Dell'Isola S, Rocco M, Ruberto F, Pugliese F, d'Ettorre G, Venditti M (2020b). Superinfections in patients treated with Teicoplanin as anti-SARSCoV2 agent. Europian Journal of Clinical Investigation, el3418.

Choudhari OK, Spalgis S, Ojha UC (2020). Cotrimoxazole as adjuvant therapy in critical ill COVID 19 patients. Journal of Ideas in Health, 3, 196-197.

Gough EK, Bourke CD, Berejena C, Shonhai A, BwakuraDangarembizi M, Prendergast AJ, Manges AR (2020). Strain-level analysis of gut-resident pro-inflammatory viridans group Streptococci suppressed by long-term cotrimoxazole prophylaxis among HIV-positive children in Zimbabwe. Gut Microbes, 11, 1104-1115.

Jean S, Hsueh P (2020). Old and re-purposed drugs for the treatment of COVID-19. Expert Review of Antiinfective Therapy, 18, 843-847.

Kadhim SS, Al-Windy SA, Al-Nami MS, Al-kuraishy HM, Al-Gareeb AI (2019). Possible Role of Statins on the Inflammatory Biomarkers in Patients With Periodontal Disease: A Cross-Sectional Study. Dental Hypotheses, 10, 70-75.

Khalil MAF, Moawad SS, Hefzy EM (2019). In vivo activity of co-trimoxazole combined with colistin against
Acinetobacter baumannii producing $\mathrm{OXA}-23$ in a Galleria mellonella model. Journal of Medical Microbiology, 68, 52-59.

Park HB, Wei Z, Oh J, Xu H, Kim CS, Wang R, Wyche TP, Piizzi G, Flavell RA, Crawford JM (2020). Sulfamethoxazole drug stress upregulates antioxidant immunomodulatory metabolites in Escherichia coli. Nature Microbiology, 5, 1319-1329.

Petersen FC, Dahle UR, Nicolau B, Casals-Pascual C (2020). COVID-19: Looking Into the Overlooked. Frontiers in Molecular Biosciences, 7, 165.

Quadery R, John T, Samuel T, Ramanna S, Chattopadhyay G, Malapanjudi C, Sodha A, Lawrence R, Dutta S, Varney V (2020a). Improved Outcomes with Trimethoprim or Cotrimoxazole in Patients with Severe COVID-19: A District Hospital Experience. Available at SSRN: https://ssrn.com/abstract=3626443.

Quadery R, John T, Samuel T, Ramanna S, Chattopadhyay G, Medveczky T, Malapanjudi C, Sodha A, Lawrence R, Dutta S, Varney V (2020b). The beneficial effects of oral trimethoprim or cotrimoxazole in patients with severe COVID-19: A case series. Preprint | SSRN | ID: ppcovidwho-1470.

Sathyamoorthy N, Chintamaneni PK, Chinni S (2020). Plausible role of combination of Chlorpromazine hydrochloride and Teicoplanin against COVID-19. Medical Hypotheses, 144, 110011.

Zhang B, Zhou X, Qiu Y, Song Y, Feng F, Feng J, Song Q, Jia Q, Wang J (2020). Clinical characteristics of 82 cases of death from COVID-19. PLoS One, 15, e0235458.

\section{How to cite this article?}

Al-Kuraishy HM, Al-Gareeb AI, Al-Buhadily AK (2020).

Cotrimoxazole and teicoplanin in the management of

Covid-19: Pleiotropic effects, shadows and lights.

Current Medical and Drug Research, 4 (2), Article ID 210. 